\title{
Change in suicidal ideation and associated factors among young adults in Hong Kong from 2018 to 2019: a latent transition analysis
}

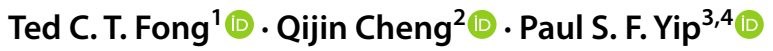

Received: 28 April 2021 / Accepted: 10 November 2021 / Published online: 24 November 2021

(c) The Author(s), under exclusive licence to Springer-Verlag GmbH Germany 2021

\begin{abstract}
Purpose Suicide in young adulthood is a vital public health concern. There have been few systematic studies that examined the prevalence and transition of suicidal ideation classes in young adults and the factors associated with the transition.

Methods This two-wave survey recruited 1269 young adults (Mean age $=24.0$ years, $70.1 \%$ males) in Hong Kong. The participants completed the Suicidal Ideation Attributes Scale, Chinese Health Questionnaire, and help-seeking behaviors in 2018 and 2019. Latent profile analysis was used to classify the participants into latent classes of suicidal ideation with substantive checking under the 3-step approach. Latent transition analysis with measurement invariance examined the transition among the latent classes from 2018 to 2019 and the associations with help-seeking behaviors and change in psychological distress. Results Three latent classes of suicidal ideation were identified and over two-thirds of the participants belonged to the Lowrisk class with minimal suicidal ideation. The prevalence of the High-risk class dropped from 10.2 to $7.2 \%$, while that of the Moderate-risk class remained stable from 2018 to 2019. Increases in psychological distress predicted higher probabilities to remain at or transit into more risky classes. Engagement in help-seeking behaviors showed differential transitions in suicidal ideation across time.

Conclusion The findings suggest three latent classes of suicidal ideation with distinct profiles among the young adults. Deterioration in psychological distress showed a temporal effect on worsening of suicidal ideation over a 1-year period. Future studies should elucidate the comparative effectiveness of help-seeking behaviors via online and offline channels.
\end{abstract}

Keywords Help-seeking behaviors $\cdot$ Latent class $\cdot$ Person-centered approach $\cdot$ Psychological distress $\cdot$ SIDAS

Paul S. F. Yip

sfpyip@hku.hk

Ted C. T. Fong

ttaatt@hku.hk

Qijin Cheng

qcheng@cuhk.edu.hk

1 Centre on Behavioral Health, The University of Hong Kong, Hong Kong, China

2 Department of Social Work, The Chinese University of Hong Kong, Hong Kong, China

3 The Hong Kong Jockey Club Centre for Suicide Research and Prevention, The University of Hong Kong, 2/F, The HKJC Building for Interdisciplinary Research, 5 Sassoon Road, Pokfulam, Hong Kong, China

4 Department of Social Work and Social Administration, The University of Hong Kong, Hong Kong, China

\section{Introduction}

Suicide remains a significant public health issue in countries worldwide [1, 2]. Increasing rates of deliberate self-harm have been found among young adults from 2007 to 2016 [3] and non-fatal self-harm has been linked with higher suicide rates and increased suicidal behavior [4]. In Hong Kong, the suicide rate was 14.2 per 100,000 in 2015 with a higher suicide rate for males than females [5] and a local study among 997 adults found a prevalence of $30.9 \%$ for lifetime suicidal ideation [6]. Despite a steady increase in the suicide rate along with age, suicide constitutes the fourth leading cause of death among young adults aged between 18 and 29 years in 2019 [7]. Suicide in young adulthood (aged between 18 and 35 years old) is a global challenge with enormous longterm impacts which requires empirical research for in-depth knowledge to mitigate the problem.

Based on the theoretical foundation of the ideation-toaction framework [8], the Interpersonal Theory of Suicide 
[9] identifies distinct phases of suicidal ideation. This theory proposes that development of suicidal ideation results from experiences of perceived burdensomeness and thwarted belongingness and an individual's capability for suicide is essential to progress from ideation to action [10]. A metaanalytic study [11] has provided support for this specific ideation-to-action framework. Suicidal ideation is a necessary condition for a suicide to occur [12] and development and escalation of suicidal ideation is a crucial intermediate step in the transition toward suicide attempts [13].

Latent class analysis and latent profile analysis are person-centered analytic techniques that focus on identifying latent subgroups within a sample based on a certain set of variables [14]. The person-centered approach differs from conventional variable-centered approach, such as factor analysis, in that the former allows finer-grained details on the potential heterogeneity in the sample [15]. Previous studies $[16,17]$ conducted latent class analysis to classify participants into latent classes of suicidal ideation in empirical samples. However, latent class analysis was cross sectional in nature and could not examine the temporal change in suicidal ideation.

Latent transition analysis (LTA) is a longitudinal analytic technique that estimates the prevalence and transition among latent classes across time [18]. This approach is useful for modeling the transition among the latent classes while allowing for likely misclassification errors and associated factors [19]. Although LTA has been applied in research on substance use and depression [20,21], previous studies [6, 22] have not applied LTA to investigate transition in suicidal ideation across time. In view of this research gap, the first objective of the present study was to apply LTA to evaluate the latent classes of suicidal ideation and examine the stability and transition among young adults in Hong Kong over one year.

Existing literature has pointed to various risk factors for suicide, such as stressful life events [23], isolation and loneliness [24], substance abuse [25], and psychological distress [26]. A meta-analysis of 50 years of research on suicidality [27] suggests that previous studies on risk factors are homogeneous and there have been mixed findings on the temporal associations between psychological distress and suicidal ideation [28, 29]. The existing studies fail to provide valuable information that predicts in what conditions a young adult will transit from distress to suicidal ideation. This calls for further studies to examine the change in suicidal ideation and the temporal association with psychological distress. The second objective of this study was to investigate the associations between transition in suicidal ideation and changes in psychological distress. We hypothesized changes in psychological distress would be significantly associated with transition toward higher suicidal risks.
Help-seeking behavior is a known protective factor among young adults with suicidal ideation $[30,31]$ and can be categorized into formal and informal help offered via offline or online channels. Presence of perceived mental health stigma among the young adults [32-34] makes it difficult for helping professionals to engage with this subpopulation. Although formal help is more effective for those with high suicidal ideation, young adults who have suicidal thoughts tend to seek informal help over formal help. Recent studies [35-37] have explored the feasibility of online help-seeking via the internet. These possibilities call for an evaluation on the comparative effectiveness of various help-seeking behaviors across informal/formal sources [38, 39] and offline/online channels [40, 41]. In view of this, the third objective of this study was to compare the transition pattern in suicidal ideation among individuals with different help-seeking behaviors.

\section{Materials and methods}

\section{Study design and procedures}

The study sample originated from an online survey of a youth emotional support (Open Up) project for the young people [42]. Two waves of data were collected in the survey in 2018 and 2019. With reference to previous literature [43-45], young adulthood referred to the age range of 18-35 years old in the present study. The inclusion criteria were young adults who were aged between 18 and 35 years old, resided in Hong Kong, and could understand written Chinese. The study participants were recruited through different platforms: bulk emails to staff and students of the local universities, poster displays to three non-governmental organizations, and online promotion means, such as webpage and Facebook. Ethical approval was obtained from the Human Research Ethics Committee of the University of Hong Kong prior to the start of the study (Reference number: EA1709039).

The participants provided voluntary informed consent before completing a self-report online questionnaire on suicidal ideation, mental well-being, and help-seeking behaviors. The questionnaire took about $10 \mathrm{~min}$ to complete. All participants were eligible to enter a lucky draw for a tablet that was worth approximately 3000 Hong Kong dollar (USD\$385) after survey completion in each wave. Participation in the survey was entirely voluntary and all of the information provided by the respondents was kept strictly confidential. Contact details of emotional support hotline and online support services were provided upon the completion of the survey as references for the participants to seek help in case they felt distressed. 


\section{Participants}

The first wave of the online survey was completed by 1352 participants from January to July 2018. A total of 83 participants were aged below 18 years old and were excluded in the present study, resulting in a final sample of 1269 young adults for subsequent data analysis. More than half of the sample were males $(70.1 \%)$ and aged between 18 and 24 years old $(60.4 \%)$. The mean age of the sample was 24.0 years $(\mathrm{SD}=4.8)$. The majority of the participants attained post-secondary education levels (86.3\%), such as higher diploma, associate and undergraduate degrees, and were students currently (74.2\%). The demographic questions were taken from a previously validated survey among young people in Hong Kong [46]. Pilot testing of the questionnaire was conducted in a small group of 20 undergraduates in a local university to ensure the acceptability and appropriateness of the questionnaire. A total of 822 respondents completed the self-report questionnaire on suicidal ideation and mental well-being at the follow-up assessment from July 2019 to October 2019.

\section{Measures}

The present study adopted the Suicidal Ideation Attributes Scale (SIDAS) [47] as a brief assessment of the severity of suicidal ideation in 2018 and 2019. This 5-item instrument measures the frequency of suicide thoughts, uncontrollability of the thoughts, closeness to suicide attempt, distress brought by the thoughts, and their interference on daily activities of the participants over the past four weeks. The five items are rated on an 11-point (0-10) anchored format and summed to produce the total score (theoretical range $=0-50$ ) with higher scores indicating greater ideation. The original developers [47] suggested the following cutoff scores for classification into three categories: No ideation (total score $=0$ ), Low ideation (total score $=1-20$ ), and High ideation (total score $=21-50$ ). The SIDAS has demonstrated good validity and reliability in samples of Australian adults and Chinese young adults [48]. Good levels of internal consistency were found in the present study $(\alpha=0.84-0.90)$.

Psychological distress was measured in 2018 and 2019 by the Chinese Health Questionnaire (CHQ) [49]. This 12-item scale was developed from the General Health Questionnaire [50] for screening of minor psychiatric morbidity. The CHQ integrates items on somatization and assesses the occurrence of mental health symptoms, such as headache, insomnia, loss of confidence, and hopelessness over the past four weeks. Items are rated on a 4-point Likert format from 0 ("Not at all") to 3 ("Much more than usual"). The total CHQ score had a theoretical range from 0 to 36 with higher scores denoting greater severity. Good levels of internal consistency were found for the CHQ ( $\alpha=0.88-0.89)$ in the present study.

The participants were asked about their help-seeking behaviors over the past four weeks as a result of distressing issues or life difficulties. Engagement in help-seeking behaviors was inquired in the following ten sources under the four categories: offline informal (family members, friends/classmates/colleagues, and partner), offline formal (guidance teachers, medical professionals, such as doctors/ nurses, social workers/counsellors, religious personnel, such as priests/pastors), online informal (online friends), and online formal (hotline support and online social service). Regarding the offline formal help, counseling is provided in secondary schools in Hong Kong under the comprehensive guidance and counseling programs and the guidance teachers have received specific trainings on addressing mental health issues. The religious personnel have attended relevant trainings of counseling help in the bible seminary. The participants could theoretically have sought help from more than one of the sources or have not sought help from any of the sources.

\section{Data analyses}

Participants' responses on suicidal ideation in 2018 and 2019 were analyzed under both the variable-centered and person-centered approaches. In the variable-centered approach, the five SIDAS items were analyzed by confirmatory factor analysis (CFA) under robust maximum likelihood estimator. Model fit was evaluated using the following criteria on approximate fit indices [51]: comparative fit index $(\mathrm{CFI}) \geq 0.95$ and root-mean-square error of approximation $($ RMSEA $) \leq 0.06$.

In the person-centered approach, latent profile analysis (LPA) was performed to identify underlying classes of suicidal ideation separately in 2018 and 2019. LPA is a variant of latent class analysis for the continuous indicators of SIDAS items in the present study [52]. LPA models with increasing number of latent classes were estimated in Mplus 8.4 [53]. Model fit was evaluated using the Bayesian information criterion (BIC) [54] with a lower value of at least 10 denoting a better fit. Item means plot describes the profiles of the latent classes and entropy represents the classification quality of the model with high values $(>0.8)$ denoting adequate classification. The number of latent classes in the sample was determined based on the BIC, entropy, and substantive checking. Substantive checking of the latent classes [55] was performed with reference to demographic characteristics, psychological distress, and help-seeking behaviors as auxiliary variables under the 3-step approach [56]. Follow-up post hoc tests examined the pairwise comparisons between latent classes with Bonferroni adjustment. 
Latent transition analysis (LTA) was then conducted to evaluate the change in the class prevalence and transition probabilities among the latent classes from 2018 to 2019 [57]. The assumption of measurement invariance in LTA was tested by comparing models with and without equal constraints on the item means. Longitudinal measurement invariance preserves the same item means for the latent classes over time and permits a straightforward interpretation of the transition probabilities [19]. Parsimony of the LTA models was compared to the longitudinal CFA models in terms of BIC. The matrix of transition probabilities depicts how likely a participant would shift from one class in 2018 to another class in 2019 or remain in the same class over time.

Potential effects of demographic covariates (age, gender, education level, and student status) on transitions in suicidal ideation were tested in the LTA model. Transition probabilities in suicidal ideation were examined separately across gender (males and females) and age groups (18-24 years old and 25-35 years old). Change in psychological distress was used to predict the transitions in suicidal ideation classes after controlling for baseline distress in the LTA model. The effects were evaluated in terms of the transition probabilities and the odds ratios (OR). Transition in suicidal ideation was analyzed with reference to the help-seeking behaviors by estimating the baseline prevalence and transition probabilities for at-risk participants with different help-seeking behaviors. Missing data were handled via the use of fullinformation maximum likelihood under the missing at random assumption [58].

\section{Results}

\section{Latent profiles of suicidal ideation}

The 1-factor CFA model provided an adequate fit to the 2018 data $\left(\chi^{2}=14.4, \mathrm{df}=5, p=0.01, \mathrm{CFI}=0.99\right.$, and RMSEA $=0.038)$. All five items had strong loadings $(\lambda=0.70-0.91, p<0.01)$ on the overall factor. Similar model fit was found for the 1-factor CFA model $\left(\chi^{2}=18.5, \mathrm{df}=5\right.$, $p<0.01, \mathrm{CFI}=0.977$, and RMSEA $=0.057)$ in the follow-up data in 2019 with significant factor loadings $(\lambda=0.40-0.90$, $p<0.01)$. These results supported a 1 -factor structure for suicidal ideation in the SIDAS.

The 1-class and 2-class LPA models provided inferior fits to the 2018 and 2019 data than the 1-factor CFA model with substantially higher BICs (Table 1). The 3-class LPA model provided a substantially lower BIC than the CFA model and 2-class LPA model in both 2018 and 2019 with high entropy (>0.90). In the 3-class LPA model, over two-thirds (68.4\%) of the sample belonged to the Low-risk class who showed minimal levels of suicidal ideation (item means $<0.7$ out of
Table 1 Fit indices of the CFA, LPA, and LTA models for suicidal ideation in 2018 and 2019

\begin{tabular}{llllll}
\hline Model & $\#$ & loglikelihood & AIC & BIC & Entropy \\
\hline 2018: $(N=1269)$ & & & & & \\
1-factor CFA & 15 & $-12,724.3$ & 25,479 & 25,556 & - \\
1-class LPA & 10 & $-14,577.8$ & 29,176 & 29,227 & - \\
2-class LPA & 16 & $-12,857.9$ & 25,748 & 25,830 & 0.958 \\
3-class LPA & 22 & $-12,343.9$ & 24,732 & 24,845 & 0.935 \\
4-class LPA & 28 & $-12,036.6$ & 24,129 & 24,273 & 0.961 \\
2019: $(N=822)$ & & & & & \\
1-factor CFA & 15 & -7676.8 & 15,384 & 15,454 & - \\
1-class LPA & 10 & -8735.0 & 17,490 & 17,537 & - \\
2-class LPA & 16 & -7689.4 & 15,411 & 15,486 & 0.968 \\
3-class LPA & 22 & -7314.9 & 14,674 & 14,778 & 0.968 \\
4-class LPA & 28 & -6989.1 & 14,034 & 14,166 & 0.974 \\
2018-2019: $(N=1269)$ & & & & & \\
1-factor CFA without & 36 & $-20,200.0$ & 40,472 & 40,657 & - \\
$\quad$ MI & & & & & \\
1-factor CFA with & 28 & $-20,244.4$ & 40,544 & 40,689 & - \\
$\quad$ MI & & & & & \\
3-3 class LTA with- & 48 & $-19,592.7$ & 39,282 & 39,529 & 0.843 \\
out MI & & & & & \\
3-3 class LTA with & 33 & $-19,649.6$ & 39,365 & 39,535 & 0.850 \\
MI & & & & & \\
\hline
\end{tabular}

CFA confirmatory factor analysis, LPA latent profile analysis, LTA latent transition analysis, \# number of free parameters, AIC Akaike information criterion, $B I C$ Bayesian information criterion, $M I$ measurement invariance

the $0-10$ scale) across the five SIDAS items on frequency, uncontrollability, associated distress, and interference of suicidal thoughts, and closeness to suicide attempt. In Fig. 1, around one-fourth $(22.1 \%)$ of the sample were classified into the Moderate-risk class with non-trivial degrees of suicidal ideation (item means $=1.8-3.5$ ). Around one-tenth $(9.5 \%)$ of the sample belonged to the High-risk class with high degrees of suicidal ideation (item means $=5.1-6.8$ ). The three latent classes showed high posterior classification probabilities $(0.94-0.98)$ in the LPA model.

\section{Comparison among the latent classes}

Table 2 displays the profiles comparison among the three latent classes in 2018. There were no significant class differences in gender, age, and student status ( $p=0.48-0.77)$ and less educated participants were more likely to belong to the High-risk class $(p<0.01)$. There were significant class differences $(p<0.01)$ in the total SIDAS score and psychological distress with increasing severity from the Low-risk class to the High-risk class. Participants in High-risk or Moderate-risk classes showed more frequent help-seeking behaviors than the Low-risk class except for offline informal help. Besides, the High-risk class showed a significantly 
Fig. 1 Profiles of the three latent classes in 3-class latent profile analysis model in 2018

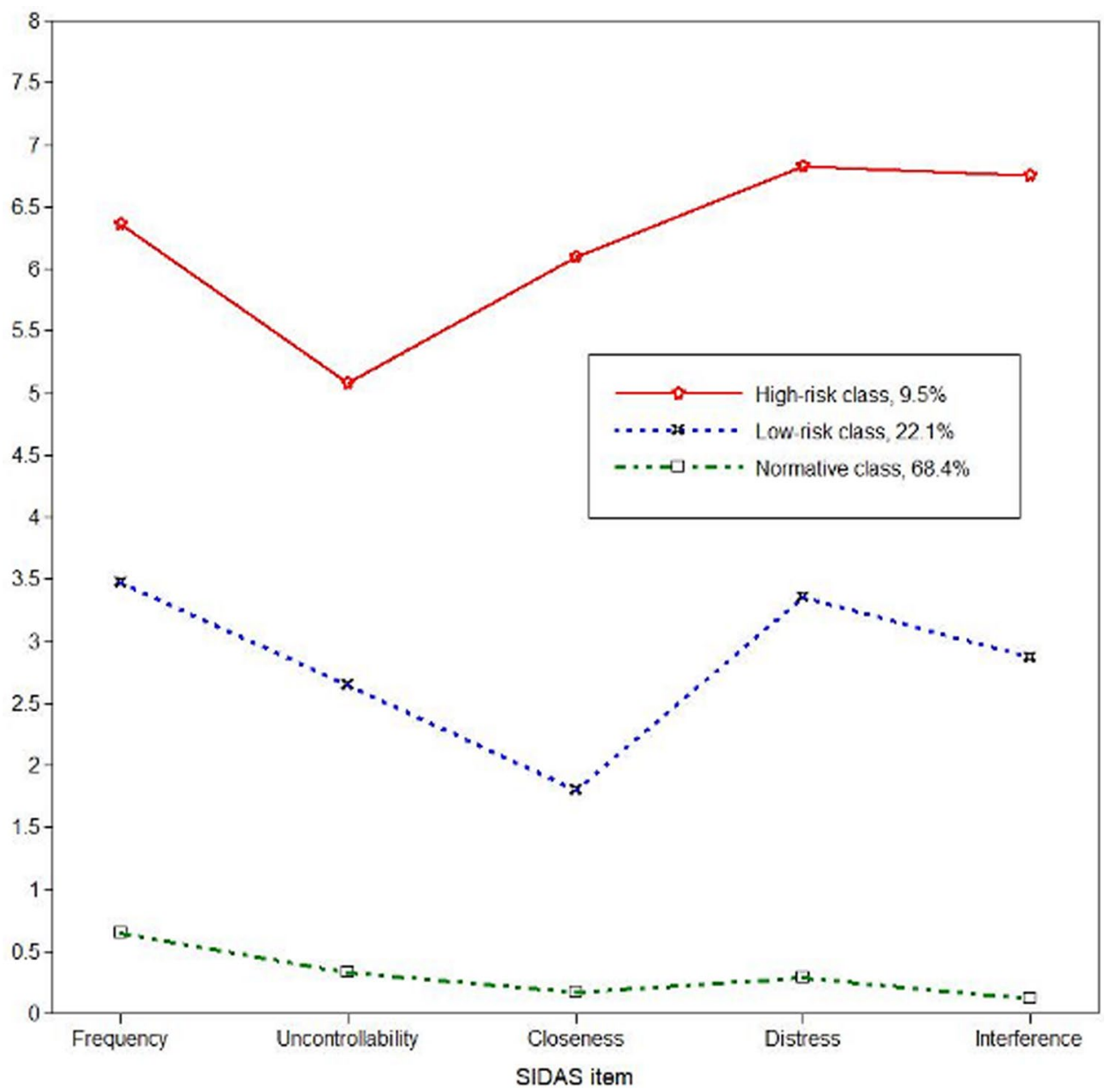

Table 2 Profiles comparison of the three latent classes of suicidal ideation under latent profile analysis in 2018

\begin{tabular}{|c|c|c|c|c|c|}
\hline Variables & $\begin{array}{l}\text { High-risk class (Class } 1) \\
(N=120) 9.5 \% \\
N(\%)\end{array}$ & $\begin{array}{l}\text { Moderate-risk class (Class } \\
\text { 2) }(N=281) 22.1 \% \\
N(\%)\end{array}$ & $\begin{array}{l}\text { Low-risk class (Class } 3) \\
(N=868) 68.4 \% \\
N(\%)\end{array}$ & $p$ & $\begin{array}{l}\text { Post hoc } \\
\text { class com- } \\
\text { parison }\end{array}$ \\
\hline Gender-female & $31(26)$ & $95(34)$ & $253(29)$ & 0.48 & \\
\hline Post-secondary education & $90(75)$ & $232(83)$ & $774(89)$ & $<0.01 *$ & $1<3$ \\
\hline Student status & $90(75)$ & $200(71)$ & $652(75)$ & 0.51 & \\
\hline Variables & Mean (SE) & Mean (SE) & Mean (SE) & $p$ & \\
\hline Age & $24.0(0.44)$ & $23.8(0.30)$ & $24.0(0.17)$ & 0.77 & \\
\hline Total SIDAS score & $31.1(2.30)$ & $14.1(1.23)$ & $1.55(0.18)$ & $<0.01 *$ & $1>2>3$ \\
\hline Psychological distress & $18.8(0.58)$ & $14.0(0.36)$ & $8.66(0.19)$ & $<0.01^{*}$ & $1>2>3$ \\
\hline \multicolumn{6}{|l|}{ Help-seeking behaviors } \\
\hline Offline informal & $0.36(0.03)$ & $0.36(0.02)$ & $0.36(0.01)$ & 0.97 & \\
\hline Offline formal & $0.20(0.03)$ & $0.08(0.01)$ & $0.05(0.01)$ & $<0.01 *$ & $1>2>3$ \\
\hline Online informal & $0.17(0.04)$ & $0.11(0.02)$ & $0.04(0.01)$ & $<0.01^{*}$ & $(1,2)>3$ \\
\hline Online formal & $0.12(0.02)$ & $0.03(0.01)$ & $0.01(0.01)$ & $<0.01^{*}$ & $1>2>3$ \\
\hline
\end{tabular}

$N=1269 ; * p<0.01$; Chi-square test statistics for equality tests of means of continuous variables across classes derived via the BCH procedure with 2 degrees of freedom 
greater likelihood $(p<0.01)$ to seek for formal help than the Moderate-risk class.

Although the 4-class LPA model showed the lowest BICs out of all measurement models, the High-risk class (item means $=6.8-8.9$ ) only had a prevalence of 3.8\%. In Supplemental Fig. S1, there were two overlapping classes with nonsignificant differences $(p>0.01)$ in four of the five SIDAS items. Supplemental Table S1 did not reveal significant differences between these two classes in most of the study variables. These results suggested a lack of clear substantive distinction among the four derived classes in the 4-class LPA model and provided support for the 3-class LPA model as the optimal model for the present sample.

\section{Associations between participant attrition and the latent classes}

Attrition analysis revealed no significant difference between the completers $(N=822)$ and dropouts $(N=447)$ in terms of demographic characteristics $(p=0.09-0.87)$ and helpseeking behaviors $(p=0.19-0.79)$. Those who dropped out of the study displayed significantly greater levels of suicidal ideation and psychological distress (Cohen $d=0.43-0.72$, $p<0.01)$ at baseline. The attrition rate of the participants in the High-risk and Moderate-risk classes (61.5\% and 50.7\%) was significantly higher $\left(\chi^{2}=96.0, \mathrm{df}=2, p<0.01\right)$ than that in the Low-risk class (26.4\%).

\section{Transition in suicidal ideation from 2018 to 2019}

The 1-factor longitudinal CFA model with measurement invariance showed acceptable fit to the 2018 and 2019 data $\left(\chi^{2}=115.1, \mathrm{df}=37, p<0.01, \mathrm{CFI}=0.971\right.$, and RMSEA $=0.041)$. The 3-3 class LTA models displayed substantially lower BICs than the 1-factor longitudinal CFA models (Table 1). The LTA model with measurement invariance estimated 15 fewer parameters with equal SIDAS item means across time for the three latent classes. This LTA model did not show a poorer fit with $\Delta \mathrm{BIC}=6.5<10$ and supported longitudinal measurement invariance of the latent classes of suicidal ideation across time.

The prevalence of and transition probabilities among the three suicidal ideation classes from 2018 to 2019 are presented in Table 3. The prevalence of the High-risk class decreased from 10.2 to $7.2 \%$, while the prevalence of the Moderate-risk class remained stable (17.3-17.6\%). Compared to the Low-risk class, the High-risk and Moderaterisk classes displayed lower temporal stability with nearly two-thirds of them (64.8-65.8\%) transiting into other classes in 2019. Age, gender, education level, and student status did not have any significant effects $\left(\chi^{2}=0.75-6.61, \mathrm{df}=4\right.$, $p=0.16-0.94)$ on the transition pattern. Supplementary Table $\mathrm{S} 2$ reports the transition probabilities among the three
Table 3 Transition model of suicidal ideation classes from 2018 to 2019 and effects of changes in psychological stress on the transition

\begin{tabular}{llll}
\hline \multicolumn{4}{c}{ Suicidal ideation class } \\
\cline { 2 - 4 } & High-risk & Moderate-risk & Low-risk \\
\hline Prevalence & & & \\
Baseline (2018) & $10.2 \%$ & $17.3 \%$ & $72.5 \%$ \\
Follow-up (2019) & $7.2 \%$ & $17.6 \%$ & $75.2 \%$ \\
Transition probabilities & & & \\
$2018 \rightarrow$ 2019 & High-risk & Moderate-risk & Low-risk \\
High-risk class & 0.352 & 0.361 & 0.287 \\
Moderate-risk class & 0.128 & 0.342 & 0.530 \\
Low-risk class & 0.019 & 0.110 & 0.871 \\
$\Delta$ CHQ $=-1$ SD decrease $\rightarrow+1$ SD increase in 2018-2019 \\
2018 $\rightarrow$ 2019 & High-risk & Moderate-risk & Low-risk \\
High-risk class & $\mathbf{0 . 4 0 3 ~} \rightarrow \mathbf{0 . 5 4 3}$ & $0.319 \rightarrow 0.210$ & $0.278 \rightarrow 0.247$ \\
Moderate-risk class & $\mathbf{0 . 0 2 7} \rightarrow \mathbf{0 . 1 6 6}$ & $\mathbf{0 . 3 0 4} \rightarrow \mathbf{0 . 5 0 3}$ & $0.669 \rightarrow 0.331$ \\
\multicolumn{5}{l}{ Low-risk class } & $\mathbf{0 . 0 0 2} \rightarrow \mathbf{0 . 0 2 6}$ & $\mathbf{0 . 0 8 5} \rightarrow \mathbf{0 . 1 6 2}$ & $0.913 \rightarrow 0.812$ \\
\hline
\end{tabular}

$N=1269 ; \mathrm{CHQ}=$ Chinese Health Questionnaire; italicized transition probabilities refer to the same class membership at both times; bolded values denote increases in the transition probabilities

suicidal ideation classes from 2018 to 2019 across gender and age subgroups. No significant differences $(p>0.05)$ were found in the transition probabilities between males and females and between the younger subgroup (aged 18-24) and older subgroup (aged 25-35).

\section{Associations between psychological distress and transition in suicidal ideation}

Although psychological distress did not change significantly $(\Delta=0.15, \mathrm{SE}=0.21, p=0.48)$ from 2018 to 2019 , there existed significant inter-individual variations (Variance $=34.88, \mathrm{SE}=1.96, p<0.01)$ in the temporal change. Controlling for demographic characteristics, baseline psychological distress in 2018 positively predicted odds of High-risk class $(\mathrm{OR}=1.33, p<0.01,95 \% \mathrm{CI}=1.26-1.39)$ and Moderate-risk class $(\mathrm{OR}=1.18, p<0.01,95 \%$ $\mathrm{CI}=1.14-1.21)$ relative to the Low-risk class. Change in psychological distress did not significantly predict transition probabilities $(B=-0.01$ to $0.05, \mathrm{SE}=0.05, p=0.39-0.90$, $\mathrm{OR}=0.99-1.05$ ) for the High-risk class in 2018. Among the Moderate-risk class in 2018, increases in psychological distress significantly predicted higher odds of transiting to the High-risk class $(\mathrm{OR}=1.12, p<0.05,95 \%$ $\mathrm{CI}=1.03-1.22)$ and lower odds of transiting to the Lowrisk class $(\mathrm{OR}=0.91, p<0.05,95 \% \mathrm{CI}=0.84-0.99)$ in 2019. Among the Low-risk class in 2018 , increases in psychological distress showed significant and positive effects on transiting to the High-risk class $(\mathrm{OR}=1.25, p<0.05$, 
$95 \% \mathrm{CI}=1.09-1.44)$ or the Moderate-risk class $(\mathrm{OR}=1.07$, $p<0.05,95 \% \mathrm{CI}=1.01-1.13$ ) in 2019 .

The bottom part of Table 3 portrays the changes in transition probabilities for participants in different latent classes with increasing versus decreasing psychological distress. Compared to participants with improved psychological distress $(\Delta=-5.76$ at Mean $-1 \mathrm{SD})$, participants with worsened psychological distress $(\Delta=+6.06$ at Mean +1 SD) in the Moderate-risk class were less likely (66.9-33.1\%) to move to the Low-risk class and more likely $(2.7-16.6 \%)$ to move to the High-risk class. Among participants in the Lowrisk class in 2018, participants with worsened psychological distress showed higher likelihoods to transit into the Highrisk class (0.2-2.6\%) or Moderate-risk class (8.5-16.2\%) compared to those with improved psychological distress.

\section{Transition pattern in suicidal ideation under different help-seeking behaviors}

Around half (48.2\%) of the sample did not report any helpseeking behaviors in 2018. The most common form of help was offline informal help (36.3\%), followed by offline formal help $(7.0 \%)$, online informal help $(6.4 \%)$, and online formal help (2.1\%). Table 4 depicts the transition probabilities among the three latent classes for participants in the Moderate-risk and High-risk classes with different help-seeking behaviors from 2018 to 2019. The majority (70.3\%) of the participants who sought offline informal help belonged to the Low-risk class. Offline informal help was linked with an improvement for $46.0 \%$ (i.e., $41.1 \%+4.9 \%$ ) and $36.3 \%$ of the High-risk and Moderate-risk classes, respectively. Online informal help resulted in an improvement for $47.1 \%$ and $25.2 \%$ of the High-risk and Moderate-risk classes, respectively. Over three-fourths (78.0\% and $81.9 \%)$ of the participants who sought formal help via offline and online sources belonged to the High-risk class. Offline formal help resulted in an improvement for $50.0 \%$ and $51.9 \%$ of the High-risk and Moderate-risk classes, respectively, while online formal help resulted in an improvement for $33.3 \%$ and $71.5 \%$ of the High-risk and Moderate-risk classes, respectively.

\section{Discussion}

The present study systematically evaluated the latent structure of suicidal ideation in a large sample of young adults in Hong Kong. The present study is among the first to apply LTA to examine transition patterns in suicidal ideation in young adults. The three latent classes showed clear separation in psychological distress and help-seeking behaviors. The additional class in the 4-class LPA model did not exhibit adequate substantive distinction with existing classes and could likely imply a spurious class without much clinical relevance. The present findings on three latent classes of suicidal ideation and their prevalence rates are consistent with previous studies using latent class analysis [16, 22, 59]. The present sample displayed a tendency for transition over stability and around two-thirds of the at-risk young adults moved to other classes. In the present study, the decrease in the prevalence of the High-risk class from 2018 to 2019 resembles the finding by Thompson et al. [22] on the transition from High-risk class to Low-risk class one year later.

From a methodological perspective, the superior fits of the LPA/LTA models over the CFA models favored the person-centered approach over variable-centered approach to capture the sample heterogeneity in suicidal ideation. The present study empirically compared LPA models with different numbers of classes via model fit and substantive checking and the current results corroborate a three-class classification of suicidal ideation into low, moderate, and high suicidal risks. The latent variable approach explicitly evaluated the classification quality via the entropy measure, took into account potential misclassification, and should be considered more robust than the existing cutoff criteria [47]. Through the use of LTA, the present findings contribute to

Table 4 Proportion of usage in 2018 and estimated transition probabilities for adolescents with various help-seeking behaviors from 2018 to 2019

\begin{tabular}{|c|c|c|c|c|c|c|}
\hline \multirow{2}{*}{$\begin{array}{l}\% \text { of usage } \\
\text { Transition probabilities }\end{array}$} & \multicolumn{3}{|c|}{ Offline informal (36.3\%) } & \multicolumn{3}{|c|}{ Online informal $(6.4 \%)$} \\
\hline & High-risk & Moderate-risk & Low-risk & High-risk & Moderate-risk & Low-risk \\
\hline High-risk class & 0.540 & 0.411 & 0.049 & 0.529 & 0.369 & 0.102 \\
\hline Moderate-risk class & 0.122 & 0.515 & 0.363 & 0.265 & 0.483 & 0.252 \\
\hline$\%$ of usage & \multicolumn{3}{|c|}{ Offline formal (7.0\%) } & \multicolumn{3}{|c|}{ Online formal (2.1\%) } \\
\hline Transition probabilities & High-risk & Moderate-risk & Low-risk & High-risk & Moderate-risk & Low-risk \\
\hline High-risk class & 0.500 & 0.303 & 0.197 & 0.667 & 0.151 & 0.182 \\
\hline Moderate-risk class & 0.293 & 0.188 & 0.519 & 0.191 & 0.094 & 0.715 \\
\hline
\end{tabular}

Transition probabilities in bold refer to transiting to a latent class with lower suicidal risk 
a deeper understanding of the risk and protective factors of the transition in suicidal ideation.

Compared to previous studies [26, 28, 29], the present analysis provided more fine-grained results on the associations between psychological distress and suicidal ideation. Among the Moderate-risk and Low-risk classes, young adults who experienced increased psychological distress were more likely to report heightened suicidal ideation. According to the ideation-to-action framework [11], such a transition to the High-risk class could contribute to subsequent progression toward suicidal behaviors [13] when combined with an individual's capability for suicide [10]. In contrast, improvements in psychological distress alone were not found to predict favorable transitions of the Highrisk class toward classes of lower risks. This discrepancy appears to suggest a unique service need of High-risk participants which require targeted and tailored suicide intervention strategies.

Offline informal help was the most common type of helpseeking behaviors and did not differentiate between the three classes of young adults. Over two-thirds of the participants receiving offline informal help belonged to the Low-risk class, suggesting that participants with minimal suicidal thoughts likely shared their daily hassles and minor issues within their social network. Offline formal help showed a consistent pattern of improvement (50-51.9\%) in the Highrisk and Moderate-risk classes. In comparison, online formal help displayed a lower and higher likelihood of improvement in the High-risk class (33.3\%) and Moderate-risk class (71.5\%), respectively. This result implies that offline formal help might be more effective in the High-risk class, whereas online formal help might be more helpful in the Moderaterisk class.

\section{Practical implications}

Amidst the current COVID-19 pandemic, social distancing procedures have been enforced to reduce human contact and control the spread of the virus. These measures have brought unintended consequences of inducing social isolation, stress, and anxiety [60], decreased access for community support, and barriers to mental health treatment [61]. These sideeffects could increase the suicidal risks and suicide attempts among the young adults [62] and have long-term impacts on the society in terms of shorter life expectancy [63]. As shown in the present study, compared to the Low-risk class, participants in the Moderate-risk class were six times as likely to transit to the High-risk class. These findings highlight a potential service gap to address the mental health distress for those in the Moderate-risk class. Early detection of the Moderate-risk class could enable timely intervention to prevent further progression into the High-risk class.
The present findings contribute to empirical knowledge toward formulating effective interventions for youth suicide prevention. Apart from standard means, such as restriction of access to means of suicide and responsible media reporting of suicide cases, other potential strategies include improved access to mental health care via a 24 -h online emotional support system and promotion of suicide prevention programs that are community based [64] or distance based [65]. Siu [66] has advocated earlier prevention strategies, such as online monitoring programs and life skills training programs, for the adolescents to help with their transition into young adulthood. A connected care model that combines online support with offline interventions could be promising in providing timely and appropriate service to the distressed individuals [36].

The internet plays a crucial role in searching for suiciderelated information and shaping subsequent suicidal behaviors for at-risk individuals [67]. Digital treatment could be considered an accessible and viable alternative to face-toface treatment for individuals who have existing distress symptoms but do not yet require specialist care. The Moderate-risk class showed a disproportionately low rate (3\%) of seeking online formal help, which likely implies service underutilization arising from the unfamiliarity of these services. To raise public awareness of online formal help, suicide prevention professionals could adopt promotion means via social media to engage the distressed young adults who are difficult to engage offline [68]. De-stigmatization campaigns (e.g., it is OK not to be OK) could be delivered to remove the barriers to seek help. Further studies can evaluate whether these measures could enhance the usage of the online formal help among young adults in need.

\section{Limitations}

The present study had a number of limitations. First, the present sample was not recruited under random sampling and the study dropouts reported significantly greater suicidal ideation and psychological distress at baseline. The baseline difference could denote potential selection bias. The disproportionate attrition rate among the three latent classes could explain the fall in the prevalence of the High-risk class and underestimate the prevalence of the Moderate-risk class in 2019. Cautions should be warranted in interpreting the overall estimates of the between-group movements before replication. Second, the current study did not control for various relevant factors, such as unemployment [5], substance abuse [25], political uncertainty [69, 70], social transmission via the internet [71, 72], and the use of social media [73]. Other factors, such as suicide stigma [74], could result in perceived barriers to seek help [34] and stressful life events [23] could lead to isolation and loneliness [24]. Further studies should 
investigate the roles of these socio-political factors [75] in the development of suicidal ideation in the young adults.

Third, the current study design consisted of only two assessments over a 1-year interval. A previous study [76] has found considerable fluctuations in suicidal ideation over shorter time frames (days). Intensive follow-up assessments are needed to elucidate the growth trajectories of suicidal ideation and timing of essential transitions, which casts doubts over the clinical relevance of the study findings. Study designs utilizing ecological momentary assessments are recommended to better capture the dynamics of changes in suicidal ideation across shorter time frames (days and weeks) and longer time frames (months and years). Fourth, the present study only offered a comparison based on the descriptive profiles of the transition pattern across different help-seeking behaviors. The overlapping nature of the help-seeking behaviors combined with the extremely sparse nature of online help-seeking behaviors made it difficult for us to incorporate them as predictors of the transition in suicidal ideation to disentangle the individual effects. Future research is required to systematically examine the effects of help-seeking behaviors on the transition in suicidal ideation and transition from suicidal ideation to suicide attempt.

\section{Conclusion}

The present study is among the first to examine the transition and stability of the three suicidal ideation classes in a large sample of young adults in Hong Kong from 2018 to 2019. Temporal linkages were found between worsened psychological distress and unfavorable transitions in suicidal ideation over one year. Differential transition patterns were found across different help-seeking behaviors and online formal help appeared to be effective for participants in the Moderate-risk class. These results put forward future directions on targeted interventions for distressed young adults and promotion of online help-seeking behaviors via social media.

Supplementary Information The online version contains supplementary material available at https://doi.org/10.1007/s00127-021-02203-9.

Acknowledgements The authors would like to express their gratitude toward The Boys' and Girls' Clubs Association, Caritas, and The Hong Kong Federation of Youths Group for their help in recruitment of participants and all respondents for their participation in the online survey. The authors would like to thank the team members of the OpenUp project for their efforts in this youth emotional support program.

Author contributions TF contributed to conceptualization, formal analysis, literature review, validation, and writing - original draft. QC was involved in conceptualization, literature review, methodology, and writing - review and editing. PY contributed to resources, conceptualization, methodology, writing — review and editing, supervision, funding acquisition.

Funding This study was supported by the Hong Kong Jockey Club Charities Trust and the General Research Fund of the Research Grants Council (GRF/HKU 17606521).

Availability of data and materials The raw data analyzed in the present study will be available by the first author without undue reservation to any qualified researcher.

Code availability The Mplus scripts will be made available from the first author to any researcher without undue reservation.

\section{Declarations}

Conflict of interest The authors declare that they have no conflicts of interest.

Ethics approval The authors assert that all procedures contributing to this work comply with the ethical standards of the relevant national and institutional committees on human experimentation and with the Helsinki Declaration of 1975, as revised in 2008. Ethics approval was obtained from the Human Research Ethics Committee of the University of Hong Kong (HREC Number = EA1709039).

Consent to participate Informed consents were obtained from all of the participants in the study.

Consent for publication All authors have approved the submission of the manuscript to the Journal. The funding organization had no role in the study design, collection, analysis and interpretation of data, or the writing of the manuscript.

\section{References}

1. Armstrong G, Vijayakumar L (2018) Suicide in India: a complex public health tragedy in need of a plan. The Lancet Public Health 3(10):e459-e460

2. Curtin SC (2020) State suicide rates among adolescents and young adults aged 10-24: United States, 2000-2018. National Vital Statistics Reports, vol 69. National Center for Health Statistics, Hyattsville, MD

3. Griffin E, McMahon E, McNicholas F, Corcoran P, Perry IJ, Arensman E (2018) Increasing rates of self-harm among children, adolescents and young adults: a 10-year national registry study 2007-2016. Soc Psychiatry Psychiatr Epidemiol 53(7):663-671. https://doi.org/10.1007/s00127-018-1522-1

4. Geulayov G, Kapur N, Turnbull P, Clements C, Waters K, Ness J, Townsend E, Hawton K (2016) Epidemiology and trends in nonfatal self-harm in three centres in England, 2000-2012: findings from the Multicentre Study of Self-harm in England. BMJ Open 6(4):e010538. https://doi.org/10.1136/bmjopen-2015-010538

5. Yang C-T, Yip PSF (2021) Changes in the epidemiological profile of suicide in Hong Kong: a 40-year retrospective decomposition analysis. China Popul Dev Stud 5(2):153-173. https://doi.org/10. 1007/s42379-021-00087-5

6. Zhang Y, Law CK, Yip PSF (2011) Psychological factors associated with the incidence and persistence of suicidal ideation. $\mathrm{J}$ Affect Disord 133(3):584-590. https://doi.org/10.1016/j.jad.2011. 05.003 
7. World Health Organization (2021) Suicide worldwide in 2019: global health estimates. Geneva

8. Klonsky ED, Saffer BY, Bryan CJ (2018) Ideation-to-action theories of suicide: a conceptual and empirical update. Curr Opin Psychol 22:38-43. https://doi.org/10.1016/j.copsyc.2017.07.020

9. Van Orden KA, Witte TK, Cukrowicz KC, Braithwaite SR, Selby EA, Joiner TE (2010) The interpersonal theory of suicide. Psychol Rev 117(2):575-600. https://doi.org/10.1037/a0018697

10. Van Orden KA, Witte TK, Gordon KH, Bender TW, Joiner TE (2008) Suicidal desire and the capability for suicide: tests of the interpersonal-psychological theory of suicidal behavior among adults. J Consult Clin Psychol 76(1):72-83. https://doi.org/10. 1037/0022-006x.76.1.72

11. Chu C, Buchman-Schmitt JM, Stanley IH, Hom MA, Tucker RP, Hagan CR, Rogers ML, Podlogar MC, Chiurliza B, Ringer FB, Michaels MS, Patros CHG, Joiner TE (2017) The interpersonal theory of suicide: a systematic review and meta-analysis of a decade of cross-national research. Psychol Bull 143(12):1313-1345. https://doi.org/10.1037/bul0000123

12. Klonsky ED, May AM, Saffer BY (2016) Suicide, suicide attempts, and suicidal ideation. In: Cannon TD, Widiger T (eds) Annual Review of Clinical Psychology, vol 12. Annual Review of Clinical Psychology. pp 307-330. https://doi.org/10.1146/annur ev-clinpsy-021815-093204

13. Paul E (2018) Proximally-occurring life events and the first transition from suicidal ideation to suicide attempt in adolescents. $\mathrm{J}$ Affect Disord 241:499-504. https://doi.org/10.1016/j.jad.2018.08. 059

14. Spurk D, Hirschi A, Wang M, Valero D, Kauffeld S (2020) Latent profile analysis: a review and "how to" guide of its application within vocational behavior research. J Vocat Behav. https://doi. org/10.1016/j.jvb.2020.103445

15. Howard MC, Hoffman ME (2018) Variable-centered, personcentered, and person-specific approaches: where theory meets the method. Organ Res Methods 21(4):846-876. https://doi.org/ $10.1177 / 1094428117744021$

16. Cho SJ (2019) Bullying victimization, negative emotionality, and suicidal ideation in korean youth: assessing latent class analysis using the manual 3-step approach. J Sch Violence 18(4):550-569. https://doi.org/10.1080/15388220.2019.1601568

17. Bernanke J, Galfalvy HC, Mortali MG, Hoffman LA, Moutier C, Nemeroff CB, Stanley BH, Clayton P, Harkavy-Friedman J, Oquendo MA (2017) Suicidal ideation and behavior in institutions of higher learning: a latent class analysis. J Psychiatr Res 95:253-259. https://doi.org/10.1016/j.jpsychires.2017.09.003

18. Lanza ST, Patrick ME, Maggs JL (2010) Latent transition analysis: benefits of a latent variable approach to modeling transitions in substance use. J Drug Issues 40(1):93-120

19. Ryoo JH, Wang C, Swearer SM, Hull M, Shi D (2018) Longitudinal model building using latent transition analysis: an example using school bullying data. Front Psychol. https://doi.org/10.3389/ fpsyg.2018.00675

20. Ulbricht CM, Dumenci L, Rothschild AJ, Lapane KL (2018) Changes in depression subtypes among men in STAR*D: a latent transition analysis. Am J Mens Health 12(1):5-13. https://doi.org/ $10.1177 / 1557988315607297$

21. Turner R, Daneback K, Skarner A (2020) Explaining trajectories of adolescent drunkenness, drug use, and criminality: a latent transition analysis with socio-ecological covariates. Addict Behav. https://doi.org/10.1016/j.addbeh.2019.106145

22. Thompson M, Kuruwita C, Foster EM (2009) Transitions in suicide risk in a nationally representative sample of adolescents. J Adolesc Health 44(5):458-463. https://doi.org/10.1016/j.jadoh ealth.2008.10.138

23. Tornblom AW, Sorjonen K, Runeson B, Rydelius PA (2020) Who is at risk of dying young from suicide and sudden violent death?
Common and specific risk factors among children, adolescents, and young adults. Suicide Life-Threat Behav 50(4):757-777. https://doi.org/10.1111/sltb.12614

24. Helm PJ, Medrano MR, Allen JJB, Greenberg J (2020) Existential isolation, loneliness, depression, and suicide ideation in young adults. J Soc Clin Psychol 39(8):641-674. https://doi.org/10.1521/ jscp.2020.39.8.641

25. Bilsen J (2018) Suicide and youth: risk factors. Front Psychiatry. https://doi.org/10.3389/fpsyt.2018.00540

26. Hawton K, Comabella CCI, Haw C, Saunders K (2013) Risk factors for suicide in individuals with depression: a systematic review. J Affect Disord 147(1-3):17-28. https://doi.org/10.1016/j. jad.2013.01.004

27. Franklin JC, Ribeiro JD, Fox KR, Bentley KH, Kleiman EM, Huang X, Musacchio KM, Jaroszewski AC, Chang BP, Nock MK (2017) Risk factors for suicidal thoughts and behaviors: a metaanalysis of 50 years of research. Psychol Bull 143(2):187-232

28. Mustanski B, Liu RT (2013) A longitudinal study of predictors of suicide attempts among lesbian, gay, bisexual, and transgender youth. Arch Sex Behav 42(3):437-448. https://doi.org/10.1007/ s10508-012-0013-9

29. Montemarano V, Troister T, Lambert CE, Holden RR (2018) A four-year longitudinal study examining psychache and suicide ideation in elevated-risk undergraduates: a test of Shneidman's model of suicidal behavior. J Clin Psychol 74(10):1820-1832. https://doi.org/10.1002/jclp.22639

30. Meyer IH, Teylan M, Schwartz S (2015) The role of help-seeking in preventing suicide attempts among lesbians, gay men, and bisexuals. Suicide Life-Threat Behav 45(1):25-36. https://doi. org/10.1111/sltb.12104

31. Lueck JA (2019) Should we activate risk perceptions in the context of suicide prevention? Examining fear appeals, help-seeking determinants, and help-seeking sources among university employees who suffer from depression. Prev Sci 20(6):884-893. https:// doi.org/10.1007/s11121-019-0979-9

32. Kosyluk KA, Al-Khouja M, Bink A, Buchholz B, Ellefson S, Fokuo K, Goldberg D, Kraus D, Leon A, Michaels P, Powell K, Schmidt A, Corrigan PW (2016) Challenging the stigma of mental illness among college students. J Adolesc Health 59(3):325-331. https://doi.org/10.1016/j.jadohealth.2016.05.005

33. Mojtabai R (2010) Mental illness stigma and willingness to seek mental health care in the European Union. Soc Psychiatry Psychiatr Epidemiol 45(7):705-712. https://doi.org/10.1007/ s00127-009-0109-2

34. Gulliver A, Griffiths KM, Christensen H (2010) Perceived barriers and facilitators to mental health help-seeking in young people: a systematic review. BMC Psychiatry 10(1):113. https://doi.org/10. 1186/1471-244X-10-113

35. Chan M, Li TM, Law YW, Wong PW, Chau M, Cheng C, Fu KW, Bacon-Shone J, Cheng QE, Yip PS (2017) Engagement of vulnerable youths using internet platforms. PLoS ONE 12(12):e0189023

36. Law YW, Kwok CL, Chan PY, Chan M, Yip P (2019) Online social work engagement and empowerment for young internet users: a quasi-experiment. J Affect Disord 250:99-107. https:// doi.org/10.1016/j.jad.2019.02.061

37. Wilks CR, Coyle TN, Krek M, Lungu A, Andriani K (2018) Suicide ideation and acceptability toward online help-seeking. Suicide Life-Threat Behav 48(4):379-385. https://doi.org/10.1111/ sltb. 12356

38. Heerde JA, Hemphill SA (2018) Examination of associations between informal help-seeking behavior, social support, and adolescent psychosocial outcomes: a meta-analysis. Dev Rev 47:44-62. https://doi.org/10.1016/j.dr.2017.10.001

39. Shumet S, Azale T, Angaw DA, Tesfaw G, Wondie M, Alennu WG, Amare T, Kassew T, Mesafint G (2021) Help-seeking preferences to informal and formal source of care for depression: a 
community-based study in northwest Ethiopia. Patient Prefer Adherence 15:1505-1513. https://doi.org/10.2147/ppa.S311601

40. Bell J, Mok K, Gardiner E, Pirkis J (2018) Suicide-related internet use among suicidal young people in the UK: characteristics of users, effects of use, and barriers to offline help-seeking. Arch Suicide Res 22(2):263-277. https://doi.org/10.1080/13811118. 2017.1334609

41. Seward AL, Harris KM (2016) Offline versus online suiciderelated help seeking: changing domains, changing paradigms. J Clin Psychol 72(6):606-620. https://doi.org/10.1002/jclp.22282

42. Yip P, Chan WL, Cheng Q, Chow S, Hsu SM, Law YW, Lo B, Ngai K, Wong KY, Xiong C, Yeung TK (2020) A 24-hour online youth emotional support: opportunities and challenges. The Lancet Regional Health Western Pacific. https://doi.org/10.1016/j. lanwpc.2020.100047

43. Petry NM (2002) A comparison of young, middle-aged, and older adult treatment-seeking pathological gamblers. Gerontologist 42(1):92-99. https://doi.org/10.1093/geront/42.1.92

44. Miller KD, Fidler-Benaoudia M, Keegan TH, Hipp HS, Jemal A, Siegal RL (2020) Cancer statistics for adolescents and young adults. Cancer J Clin 70(6):443-459. https://doi.org/10.3322/caac. 21637

45. Bratincsák A, Kimata C, Limm-Chan BN, Vincent KP, Williams MR, Perry JC (2020) Electrocardiogram standards for children and young adults using z-scores. Circ Arrhythmia Electrophysiol 13(8):e008253. https://doi.org/10.1161/CIRCEP.119.008253

46. Wong PWC, Li TMH, Chan M, Law YW, Chau M, Cheng C, Fu KW, Bacon-Shone J, Yip PSF (2015) The prevalence and correlates of severe social withdrawal (hikikomori) in Hong Kong: a cross-sectional telephone-based survey study. Int J Soc Psychiatry 61(4):330-342. https://doi.org/10.1177/0020764014543711

47. van Spijker BAJ, Batterham PJ, Calear AL, Farrer L, Christensen H, Reynolds J, Kerkhof AJFM (2014) The Suicidal Ideation Attributes Scale (SIDAS): community-based validation study of a new scale for the measurement of suicidal ideation. Suicide LifeThreat Behav 44(4):408-419. https://doi.org/10.1111/sltb.12084

48. Han J, Batterham PJ, Calear AL, Wu Y, Shou Y, van Spijker BAJ (2017) Translation and validation of the Chinese versions of the suicidal ideation attributes scale, stigma of suicide scale, and literacy of suicide scale. Death Stud 41(3):173-179. https://doi.org/ 10.1080/07481187.2016.1214633

49. Chong MY, Wilkinson G (1989) Validation of 30-item and 12-item versions of the Chinese Health Questionnaire (CHQ) in patients admitted for general health screening. Psychol Med 19(2):495-505. https://doi.org/10.1017/s0033291700012526

50. Goldberg DP, Hillier VFJPM (1979) A scaled version of the General Health Questionnaire. Psychol Med 9(1):139-145

51. Hu LT, Bentler PM (1999) Cutoff criteria for fit indexes in covariance structure analysis: conventional criteria versus new alternatives. Struct Equ Model 6(1):1-55. https://doi.org/10.1080/10705 519909540118

52. Ferguson SL, Moore EWG, Hull DM (2020) Finding latent groups in observed data: a primer on latent profile analysis in Mplus for applied researchers. Int J Behav Dev 44(5):458-468. https://doi. org/10.1177/0165025419881721

53. Muthén LK, Muthén BO (2017) Mplus user's guide. 8th edn. Muthén \& Muthén, Los Angeles

54. Raftery AE (1995) Bayesian model selection in social research. Sociol Methodol 25:111-163. https://doi.org/10.2307/271063

55. Muthén B (2003) Statistical and substantive checking in growth mixture modeling: comment on Bauer and Curran (2003). Psychol Methods 8(3):369-377. https://doi.org/10.1037/1082-989X.8.3. 369

56. Asparouhov T, Muthén B (2014) Auxiliary variables in mixture modeling: three-step approaches using M plus. Struct Equ Modeling 21(3):329-341
57. Collins LM, Lanza ST (2009) Latent class and latent transition analysis: With applications in the social, behavioral, and health sciences, vol 718. John Wiley \& Sons

58. Little RJA, Rubin DB (2014) Statistical analysis with missing data. Wiley, New York

59. Hamza CA, Willoughby $T$ (2013) Nonsuicidal self-injury and suicidal behavior: a latent class analysis among young adults. PLoS ONE. https://doi.org/10.1371/journal.pone.0059955

60. Yip PSF, Chau PH (2020) Physical distancing and emotional closeness amidst COVID-19. Crisis J Crisis Interv Suicide Prev 41(3):153-155. https://doi.org/10.1027/0227-5910/a000710

61. Reger MA, Stanley IH, Joiner TE (2020) Suicide mortality and coronavirus disease 2019-a perfect storm? JAMA Psychiat 77(11):1093-1094. https://doi.org/10.1001/jamapsychiatry. 2020.1060

62. McIntyre RS, Lee Y (2020) Preventing suicide in the context of the COVID-19 pandemic. World Psychiatry 19(2):250-251

63. Yip PSF, Zheng Y (2021) The impact of suicide on life expectancy. Crisis J Crisis Interv Suicide Prev 42(2):107-113. https:// doi.org/10.1027/0227-5910/a000695

64. Lai C, Law YW, Shum AK, Ip FW, Yip PS (2020) A community-based response to a suicide cluster: a Hong Kong experience. Crisis J Crisis Interv Suicide Prev 41(3):163-171. https:// doi.org/10.1027/0227-5910/a000616

65. Gunnell D, Appleby L, Arensman E, Hawton K, John A, Kapur N, Khan M, O'Connor RC, Pirkis J, Appleby L, Arensman E, Caine ED, Chan LF, Chang S-S, Chen Y-Y, Christensen H, Dandona R, Eddleston M, Erlangsen A, Gunnell D, HarkavyFriedman J, Hawton K, John A, Kapur N, Khan M, Kirtley OJ, Knipe D, Konradsen F, Liu S, McManus S, Mehlum L, Miller M, Moran P, Morrissey J, Moutier C, Niederkrotenthaler T, Nordentoft M, O'Connor RC, O'Neill S, Page A, Phillips MR, Pirkis J, Platt S, Pompili M, Qin P, Rezaeian M, Silverman M, Sinyor M, Stack S, Townsend E, Turecki G, Vijayakumar L, Yip PSF (2020) Suicide risk and prevention during the COVID-19 pandemic. The Lancet Psychiatry 7(6):468-471. https://doi.org/ 10.1016/S2215-0366(20)30171-1

66. Siu AMH (2019) Self-harm and suicide among children and adolescents in Hong Kong: a review of prevalence, risk factors, and prevention strategies. J Adolesc Health 64(6):S59-S64. https://doi.org/10.1016/j.jadohealth.2018.10.004

67. Hawton K, Saunders KEA, O'Connor RC (2012) Self-harm and suicide in adolescents. Lancet 379(9834):2373-2382. https:// doi.org/10.1016/s0140-6736(12)60322-5

68. Cheng Q, Shum AKY, Ip FWL, Wong HK, Yip WKK, Kam AHL, Yip PSF (2020) Co-creation and impacts of a suicide prevention video: a case study of a YouTube short film on youth suicide prevention. Crisis J Crisis Interv Suicide Prev 41:7-14

69. Hou WK, Hall BJ (2019) The mental health impact of the prodemocracy movement in Hong Kong. The Lancet Psychiatry 6(12):982. https://doi.org/10.1016/S2215-0366(19)30382-7

70. Hou WK, Hall BJ, Liang L, Li TW, Liu H, Galea S (2020) Probable Depression and suicidal ideation in Hong Kong amid massive civil unrest. Ann Epidemiol. https://doi.org/10.1016/j. annepidem.2020.09.006

71. Marchant A, Hawton K, Stewart A, Montgomery P, Singaravelu V, Lloyd K, Purdy N, Daine K, John A (2017) A systematic review of the relationship between internet use, self-harm and suicidal behaviour in young people: the good, the bad and the unknown. PLoS ONE. https://doi.org/10.1371/journal.pone. 0181722

72. Jacob N, Evans R, Scourfield J (2017) The influence of online images on self-harm: a qualitative study of young people aged 16-24. J Adolesc 60:140-147. https://doi.org/10.1016/j.adole scence.2017.08.001 
73. Nathan NA, Nathan KI (2020) Suicide, stigma, and utilizing social media platforms to gauge public perceptions. Front Psych. https:// doi.org/10.3389/fpsyt.2019.00947

74. Fong TCT, Yip PSF, Chan MYH, Ho RTH (2020) Factor structure and measurement invariance of the Stigma of Suicide ScaleShort Form among adolescents and early adults in Hong Kong. Assessment. https://doi.org/10.1177/1073191120976857

75. Yang X, Mak WWS (2020) Addressing sociopolitical determinants of mental health: an emerging challenge in Hong Kong.
The Lancet 395(10220):249-250. https://doi.org/10.1016/S01406736(20)30057-X

76. Kleiman EM, Turner BJ, Fedor S, Beale EE, Huffman JC, Nock MK (2017) Examination of real-time fluctuations in suicidal ideation and its risk factors: results from two ecological momentary assessment studies. J Abnorm Psychol 126(6):726-738. https:// doi.org/10.1037/abn0000273 